\title{
Sarcoma sinovial bifásico pulmonar. Presentación de un caso y revisión de la literatura
}

\author{
FRANCISCO JAVIER TORRES-GÓMEZ*, FRANCISCO JOSÉ-VÁZQUEZ-RAMÍREZ**, \\ FRANCISCO JAVIER TORRES-OLIVERA**
}

Biphasic synovial sarcoma of the lung. Report of a case and review of the literature

Introduction: Synovial sarcoma is an aggressive neoplasm described in several locations but is uncommon in the lung. Method: We report a case of biphasic synovial sarcoma of the lung stressing the histologic and immunohistochemical characteristics, genetics and differential diagnosis. Results: a biphasic pattern and SYT-SSX translocation were demostrated. Comments: histology and immunohistochemistry lead to diagnosis most of the times but the typical translocation is definitive.

Key words: Synovial sarcoma, lung, SYT-SSX translocation.

Resumen

Introducción: El sarcoma sinovial es una neoplasia agresiva que aun habiendo sido descrita en múltiples localizaciones, resulta sumamente infrecuente en localización pulmonar. Método: Presentamos un caso de sarcoma sinovial bifásico de localización pulmonar haciendo hincapié en sus características histológicas inmunohistoquímicas y genéticas así como en su diagnóstico diferencial. Resultados: La neoplasia mostraba un patrón bifásico bien caracterizado. El estudio genético demostró la translocación SYT-SSX. Comentarios: Si bien la histología y la inmunohistoquímica permiten en la mayoría de los casos el diagnóstico del sarcoma sinovial, es la translocación genética la que define verdaderamente esta entidad.

Palabras clave: Sarcoma, sinovial, pulmón, translocación SYT-SSX.

\section{Introducción}

A pesar de su denominación, es sumamente infrecuente encontrar un sarcoma sinovial en localización intra-articular, siendo la ubicación más común para esta infrecuente neoplasia en torno a los tejidos blandos peri-articulares, a nivel de extremidades. No obstante, muchas han sido las localizaciones en la economía corporal en las que se han descrito casos de sarcoma sinovial, muchas de ellas excepcionales, como ocurre con el pulmón. Se trata de una neoplasia agresiva asociada a mal pronóstico para la que será necesaria una terapia agresiva consistente, en la mayoría de las ocasiones, en exéresis quirúrgica amplia y adyuvancia. En casos en los que no pueda llevarse a cabo este agresivo tratamiento será frecuente asistir a recurrencias o metástasis, tempranas o tardías.

Siendo el pulmón el lugar preferente de metástasis visceral del sarcoma sinovial localizado en extremidades, será necesario descartar un origen primario en las mismas antes de asignar a aquel como origen de la neoplasia.

\section{Caso clínico}

Paciente varón de 50 años de edad, exfumador, con antecedentes clínicos de hipertensión arterial e hipercolesterolemia. El único antecedente quirúrgico reseñado fue una intervención quirúrgica sobre el pie izquierdo hacía 25 años que no parecía guardar relación con el actual

* Unidad de Anatomía Patológica. Hospital de Alta Resolución de Utrera. Sevilla.

** Servicio de Anatomía Patológica. Hospital Universitario Virgen Macarena. Sevilla. 
motivo de consulta, consistente en dolor torácico inespecífico acompañado de un cuadro respiratorio igualmente inespecífico. El paciente, en resumen, sentía cierta desazón que le incitaba a acudir a consulta. Se le realizó una radiografía anteroposterior de tórax en la que se observó una lesión ocupante de espacio en el lóbulo superior de pulmón izquierdo cuyo tamaño fue estimado en 10 x $6 \mathrm{~cm}$ de dimensiones máximas (Figura 1). El estudio posterior con TAC confirmó los hallazgos (Figura 2). Se realizó la lobectomía pulmonar correspondiente observándose, a los cortes seriados, una lesión nodular blanquecina de aspecto carnoso, discretamente estriada, en cuyo seno se observaron quistes de mínimo tamaño y en periferia varios coágulos hemáticos. La lesión, en su conjunto, se encontraba bien delimitada del parénquima circundante, ligeramente condensado (Figura 3).

El estudio histológico lesional demostró una arquitectura lobular en la que células de hábito fusiforme formaban haces entrelazados con focal disposición “en rueda de carro”(en España está extendido el uso del término "estoriforme") Los núcleos, con heterogénea hipercromasia, mostraban márgenes romos. Se observaban, de igual modo, áreas con menor densidad celular en las que se apreciaban estructuras glandulares monoestratificadas de contorno ovoideo tapizadas por epitelio cúbico en cuyas luces albergaban secreción eosinofílica. Dichas estructuras glandulares estaban rodeadas del componente fusiforme previamente descrito. El índice mitósico era moderado, observándose figuras de mitosis principalmente en el componente fusocelular (Figuras 4 y 5).

Se realizaron técnicas inmunohistoquímicas demostrándose inmunopositividad del componente glandular-tubular para citoqueratinas de amplio espectro, AME, TTF-1 y CD99 (débil). El componente fusocelular, por el contrario, mostró únicamente positividad focal para AME y s100. Ambos componentes fueron negativos frente a los marcadores c-Kit, AML y calretinina. Con la sospecha de sarcoma sinovial bifásico se efectuaron estudios moleculares (FISH y RT-PCR -reacción en cadena de la polimerasa en tiempo real-), confirmándose la translocación t $(\mathrm{x} ; 18)$ (SYT,SSX) (Figuras 6 y 7).

Se realizó un riguroso estudio de extensión en el que se descartaron focos neoplásicos en cualquier otra localización.

El paciente fue tratado con ciclos de radioterapia. No se incluyó en ciclos de quimioterapia. Se realizó seguimiento del paciente observándose a los cuatro años metástasis peri-pancreáticas del sarcoma sinovial original. El paciente permanece vivo seis años después del diagnóstico inicial sin que tengamos constancia de nuevas intervenciones terapéuticas.

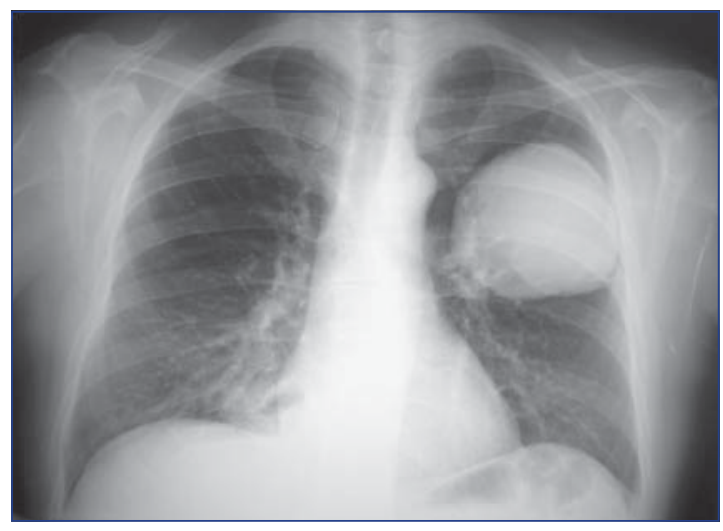

Figura 1. Sarcoma sinovial bifásico pulmonar: Rx anteroposterior de tórax.

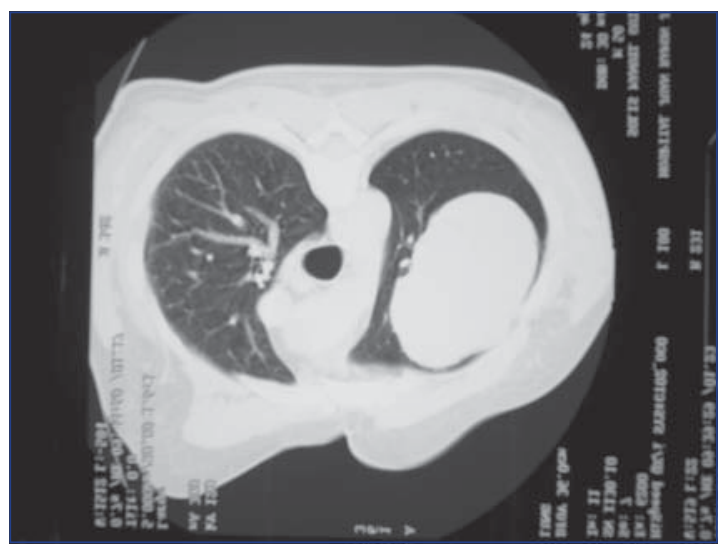

Figura 2. Sarcoma sinovial bifásico pulmonar: TAC torácico.

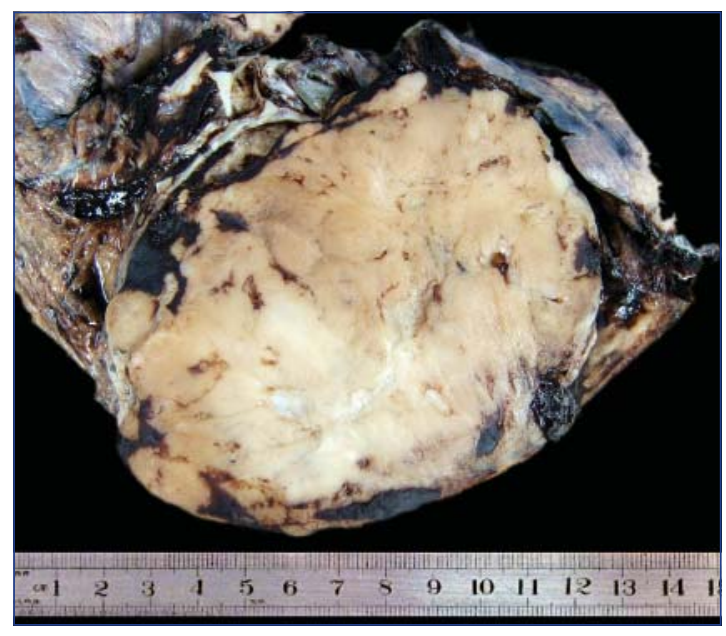

Figura 3. Sarcoma sinovial bifásico pulmonar: Imagen macroscópica. 


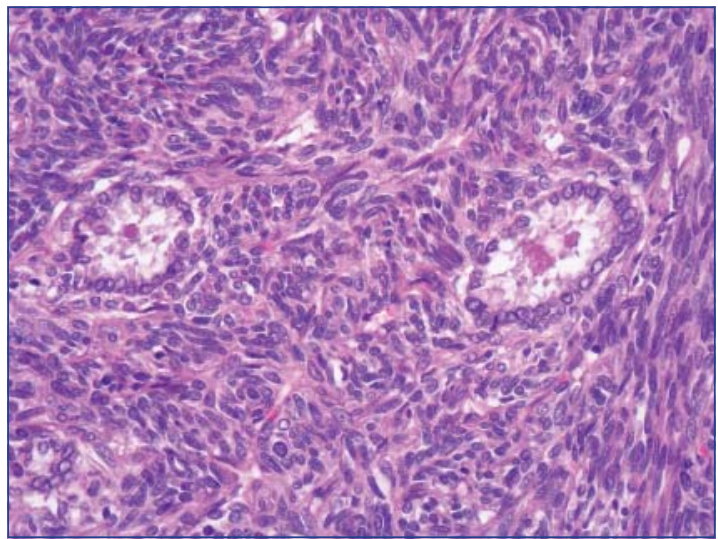

Figura 4. Sarcoma sinovial bifásico pulmonar: Detalle de estructuras glandulares. HE. 400x.

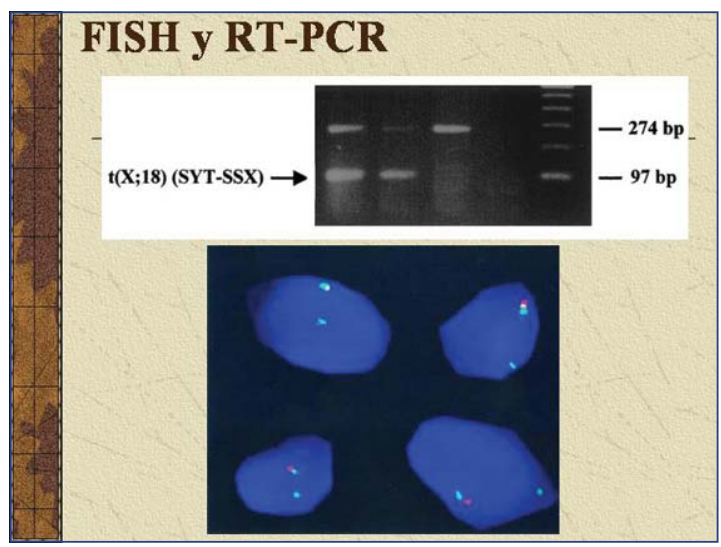

Figura 6. Sarcoma sinovial bifásico pulmonar: Translocación t $(x, 18)$ SYT-SSX. FISH y RT-PCR.

\section{Discusión}

El sarcoma sinovial es una neoplasia morfológicamente bien definida cuya localización más común reside en tejidos blandos, en la vecindad de las grandes articulaciones. Pese a su actual denominación, basada en antiguas descripciones hoy en día desfasadas, no existen evidencias de que el sarcoma sinovial se origine o diferencie a tejido sinovial y su localización extra-articular así lo atestigua; es más, estudios inmunofenotípicos y moleculares confirman las diferencias filogenéticas de la neoplasia y el tejido sinovial. No obstante, se trata de una nomenclatura bien arraigada que lucha por mantenerse vigente ${ }^{1}$.

Constituye en torno al 5-10\% de los sarcomas de partes blandas. El 90\% de los mismos se localizan en torno a grandes articulaciones (la rodilla es la más frecuente) y el 10\% muestran una localización "extraesquelética”. Hay en este punto que destacar que se ha descrito sarcoma sinovial

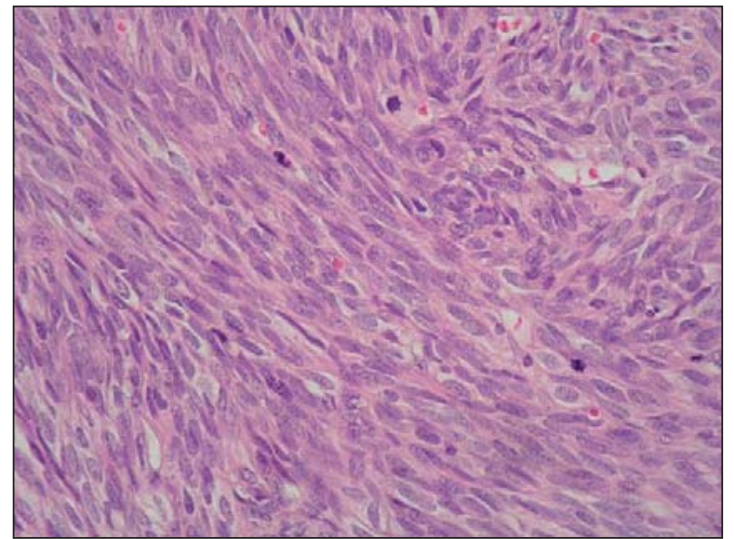

Figura 5. Sarcoma sinovial bifásico pulmonar: Detalle de componente fusocelular. Alto índice mitósico. HE. 400x.

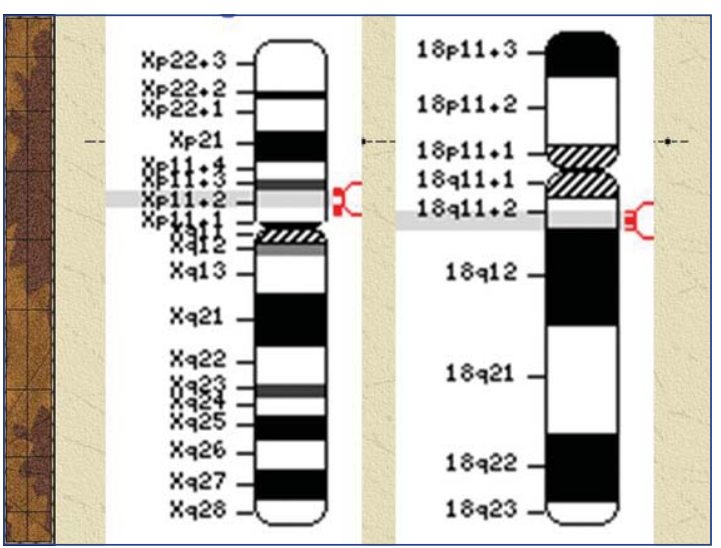

Figura 7. Sarcoma sinovial bifásico pulmonar: Cromosoma-translocación.

en prácticamente cualquier parte del organismo si bien la localización visceral, como la descrita en nuestro caso, es sumamente inusual, hecho que condiciona que muchas veces no se piense en el diagnóstico de sarcoma sinovial durante el planteamiento diagnóstico inicial. El 50\% de los sarcomas sinoviales metastatiza, siendo el pulmón el órgano más frecuentemente afectado. La supervivencia global se estima en torno al 40$50 \%$ a los 10 años.

El espectro morfológico del sarcoma sinovial es amplio, habiéndose descrito cuatro variantes o fenotipos principales: bifásico, fibroso monofásico, epitelial monofásico (el menos frecuente) y el poco diferenciado. Las características histológicas de cada uno de ellos se corresponden con su definición (el subtipo pobremente diferenciado suele estar constituido por células redondas y más que una entidad o subtipo parece corresponderse con un patrón de desdiferenciación).

Nuestro caso fue diagnosticado de sarcoma 
sinovial bifásico pulmonar. Esta variante morfológica, como su mismo nombre indica, está constituida por dos componentes bien definidos en la mayoría de los casos: un componente epitelial, consistente en células poligonales con núcleo central y citoplasma claro bien definido agrupadas en patrón sólido, nodular o glandular, y un componente fusocelular homogéneo similar al observado en el fibrosarcoma. También han sido descritas variantes celulares mixtas de ambos componentes. Los estudios estructurales, inmunohistoquímicos y moleculares han acreditado una filiación común de ambos tipos celulares.

Existen otros sarcomas que pueden exhibir una histología similar y por tanto, será necesario realizar un amplio diagnóstico diferencial; nos referimos al sarcoma epitelioide, al sarcoma de células claras, al schwannoma maligno, al schwannoma maligno glandular, al fibrosarcoma, a los carcinomas y a los carcinosarcomas.

El caso que presentamos muestra localización pulmonar. Dicha localización es infrecuente, habiéndose descrito menos de 100 casos en la literatura. El estudio de los mismos permite sacar una serie de conclusiones: es más frecuente en varones, con una media de edad de 40 años si bien la mayoría de los casos han sido descritos en una población joven, por debajo de la cuarta década, tienen preferencia por la periferia visceral, la histología es superponible a la de las localizaciones "esqueléticas" y la supervivencia global a los 5 años se sitúa en torno al 50\%. A la hora de realizar el diagnóstico diferencial tendremos que tener en cuenta neoplasias propias de la región torácica tales como el mesotelioma maligno y el leiomiosarcoma ${ }^{2-4}$.

Cuando afrontamos el diagnóstico diferencial tenemos que tener en cuenta la posibilidad de que éste no pueda realizarse con garantías sin el auxilio de determinaciones genéticas. No obstante, la morfología y la inmunohistoquímica juegan un importante papel. Para realizar el diagnóstico diferencial entre la variante bifásica, como ocurre en nuestro caso, con los carcinosarcomas, hemos de tener en cuenta la mayor atipia celular e incluso pleomorfismo exhibidos por el segundo en contraste con el primero. El schwannoma maligno glandular, por su parte, muestra elementos glandulares de tipo intestinal, incluso células mucosecretoras, ausentes en el sarcoma sinovial. Como hemos comentado, la localización pulmonar obliga a descartar los mesoteliomas malignos si bien la historia clínica y la tendencia a una presentación difusa del mesotelioma deben orientar a su diagnóstico. En cualquier caso, es conveniente realizar un sistemático estudio inmunohistoquímico (calretinina, positiva en los mesoteliomas y en el $50 \%$ de los sarcomas sinoviales de esta localización, Ber-EP4, positivo en los sarcomas sinoviales y negativo en los mesoteliomas, y WT1 negativo en sarcomas sinoviales y positivo en mesoteliomas). En casos de difícil diagnóstico siempre podrá recurrirse al estudio genético para poner de manifiesto la translocación característica ${ }^{1}$. Por último, no debemos olvidar que el pulmón se encuentra entre las principales localizaciones de destino de las metástasis de sitios primarios en extremidades; se deberá, pues, descartar esta posibilidad antes de considerar la neoplasia como primaria pulmonar.

Podemos aplicar un amplio panel de técnicas inmunohistoquímicas que nos permita afrontar los diagnósticos diferenciales; ninguna de ellas es específica para el sarcoma sinovial. La confirmación diagnóstica se realiza con técnicas moleculares, detectando la translocación $\mathrm{t}(\mathrm{X} ; 18)$ (p11,q11): SYT-SSX1, SYT-SSX2, SYT-SSX4, teniendo en cuenta que en el $10 \%$ de los casos no hay mutación. La presencia de SYT-SSX supone la fusión del fragmento 5’SYT (cromosoma 18) y del fragmento 3'SSX (cromosoma X).La proteína quimérica resultante se considera específica del sarcoma sinovial y por tanto, diagnóstica. Se cree que estas proteínas actúan como reguladores anómalos de la trascripción, dando lugar a la activación de oncogenes o a la inhibición de genes de supresión tumoral ${ }^{5-9}$.

Sacamos como conclusión que el sarcoma sinovial es una neoplasia de difícil diagnóstico, principalmente la variante monofásica. Es preciso recurrir a algoritmos diagnósticos en la mayoría de los casos. Aun así, hoy en día es imprescindible recurrir a técnicas moleculares que nos permitan detectar la translocación diagnóstica.

Detengámonos un instante en analizar los factores pronósticos del sarcoma sinovial. Independientemente de su localización, los factores pronósticos clásicos son el tamaño (peor pronóstico para neoplasias mayores de $5 \mathrm{~cm}$ ), el grado citológico, el índice mitósico (peor pronóstico si el contaje supera las 10-15 figuras de mitosis /10 campos de gran aumento), la presencia de necrosis, la radioterapia adyuvante y la propia edad adulta (se ha descrito un mejor pronóstico en niños). La incorporación de estudios moleculares ha aportado nuevos factores pronósticos en este ámbito, en concreto la presencia o ausencia de la t(x; 18). La afectación de los márgenes quirúrgicos ha demostrado igualmente tener importancia pronóstica ${ }^{1,10-12}$.

El tratamiento de esta peculiar neoplasia consiste en la exéresis quirúrgica, amplia, aso- 
ciada en la mayoría de las ocasiones a terapias adyuvantes. De hecho, se trata de un tumor quimiosensible habiéndose descrito buenas respuestas con regímenes que incluyan ifosfamida, doxorrubicina y epirrubicina ${ }^{13}$. Hoy día se está experimentando con nuevas modalidades terapéuticas en concordancia con dianas terapéuticas. Al ser sobreexpresada Bcl2 (proteína antiapoptótica) en la mayoría de los casos, terapias destinadas a su bloqueo (G3139) favorecerían la apoptosis de las células neoplásicas ${ }^{14,15}$. Algo similar ocurriría con el uso de Gefitinib contra EGFR, expresado en más de la mitad de los casos de sarcoma sinovial ${ }^{16}$. Por último, podría incluso valorarse el uso de Trastuzumab siempre que se demostrara la sobreexpresión de HER-2, presente en algunos $\operatorname{casos}^{17}$.

\section{Bibliografía}

1.- WEISS S W, GOLDBLUM J R. Synovial sarcoma. En: SW Weiss y JR Goldblum, Eds. Enzinger and Weiss's Soft Tissue Tumors (5a edición) Mosby. St Louis, MO 2008, pp 1161-82.

2.- TERASAKI H, NIKE T, HASEGAWA T, YAMADA T, SUZUKI K, KUSUMOTO M, et al. Primary synovial sarcoma of the lung: a case report confirmed by molecular detection of SYT-SSX fusion gene transcripts. Jpn J Clin Oncol 2001; 31: 212-6.

3.- LEE YF, JOHN M, EDWARDS S, CLARK J, FLOHR P, MAILLARD K, et al. Molecular classification of synovial sarcomas, leiomyosarcomas and malignant fibrous histiocytomas by gene expression profiling. Brit J Cancer 2003; 88: 510-15.

4.- SUSTER S, MORAN C A. Primary synovial sarcomas of the mediastinum. A clinicopathologic, immunohistochemical and ultrastuctural study of 15 cases. Am J Surg Pathol 2005; 29: 569-78.

5.- HOSONOT, HIRONAKA M, KOBAYASHI A, YAMASAWA H, BANDO M, OHNO S. Primary pulmonary synovial sarcoma confirmed by molecular detection of SYT-SSX1 fusion gene transcripts: a case report and review of the literature. Jap J Clin Oncol 2005; 35: 274-9.

6.- BEGUERET H, GALATEAU-SALLE F, GUILLOU L, CHETAILLEB, BRAMBILLA E, VIGNAUD J M, et al Primary intrathoracic synovial sarcoma. A clinicopatho- logic study of $40 \mathrm{t}(\mathrm{x} ; 18)$ positive cases from the French Sarcoma Group and the Mesopath Group. Am J Surg Pathol 2005; 29: 339-46.

7.- LADANYI M. Fusions of the SYT and SSXgenes in synovial sarcoma. Oncogene 2001; 20: 5755-62.

8.- YANO M, TOYOOKA S, TSUKUDA K, DOTE $\mathrm{H}$, MORIMOTO Y, OHATA N, et al. SYT-SSX fusion genes in synovial sarcoma of the thorax. Lung Cancer 2004; 44: 391-7.

9.- OLIVEIRA A, FLETCHER C. Molecular prognostication for soft tissue sarcomas: are we ready yet?. J Clin Oncol 2004; 22: 4031-4.

10.- SEGAL N H, PAVLIDIS P, ANTONESCU C R, MAKI R G, NOBLE W S, DESANTIS D, et al Classification and subtype prediction of adult soft tissue sarcoma by functional genomics. Am J Pathol 2003; 163 : 691-700.

11.- SPURREL E L, FISHER C, THOMAS J M, JUDSON I R. Prognostic factors in advanced synovial sarcoma: an análisis of 104 patients treated at the Royal Marsden Hospital. Ann Oncol 2005; 16: 437-44.

12.- FERRIS I TORTAJADA J, ORTEGA GARCÍA J A, LÓPEZ ANDREU JA, GARCÍA I CASTELL J, BERBEL TORNERO O, LLINARES RIESTRA E, et al Factores de riesgo asociados a sarcomas de partes blandas pediátricos. Rev Esp Pediat 2001; 57: 445-57.

13.- ALBRITTON K H, RANDALL R L. Prospects for targered therapy of synovial sarcoma. J Pediatr Hematol Oncol 2005; 27: 219-22.

14.- MANCUSO T, MEZZELANI A, RIVA C, FABBRI A, DAL BO L, SAMPIETRO G, et al Analysis of SYT-SSX fusion transcripts and bcl-2 expression and phosphorylation status in synovial sarcoma. Lab Invest 2000; 80: 805-13.

15.- JOYNER D E, ALBRITTON K H, BASTAR J D, RANDALL R L. G3139 antisense oligonucleotide directed against antiapoptotic bcl-2 enhances doxorubicin cytotoxicity in the FU-SY-1 synovial sarcoma cell line. J Orthop Res 2006; 24: 474-80.

16.- BLAY J Y, RAY-COQUARD I, ALBERTI L, RANCHERE D. Targeting other abnormal signaling pathways in sarcoma: EGFR in synovial sarcomas, PPAR-gamma in liposarcomas. Cancer Treat Res 2004; 120: 151-67.

17.- NUCIFORO P G, PELLEGRINI C, FASANI R, MAGGIONI M, COGGI P, PARAFIORITI A, et al. Molecular and immunohistochemical analysis of HER-2/neu oncogene in synovial sarcoma. Hum Pathol 2003; 34: 639-45.

\footnotetext{
Correspondencia a:

Dr. Francisco Javier Torres Gómez

Unidad de Anatomía Patológica

Hospital de Alta Resolución de Utrera. Sevilla.

Avda. Brigadas Internacionales s/n. 41710.

Utrera. Sevilla.

E-mail: javiertorresgomez@yahoo.es
}

Rev Chil Enf Respir 2011; 27: 203-207 